\title{
N-(3-Ethoxy-phenyl)-4-pyrrolidin-1-yl-3-trifluoromethyl-benzamide (EPPTB) prevents 3-iodothyronamine (T1AM)-induced neuroprotection against kainic acid toxicity
}

\author{
Elisa Landucci ${ }^{\mathrm{a}}$, Manuela Gencarelli ${ }^{\mathrm{b}}$, Costanza Mazzantini ${ }^{\mathrm{b}}$, Annunziatina Laurino ${ }^{\mathrm{b}, 1}$, \\ Domenico Edoardo Pellegrini-Giampietro ${ }^{\mathrm{a}}$, Laura Raimondi ${ }^{\mathrm{b}, *}$
}

${ }^{a}$ Dept. of Health Sciences, Section of Pharmacology, University of Florence, 50139, Florence, Italy

${ }^{\mathrm{b}}$ Dept. of Neurology, Psychology, Drug Sciences and Child Health, Section of Pharmacology, University of Florence, 50139, Florence, Italy

\section{A R T I C L E I N F O}

\section{Keywords:}

3-iodothyroanmine

T1AM

3-iodothyroacetic acid

TA1

Trace amine associated receptor isoform 1

TAAR1

Protein kinase B

AKT

Protein kinase A

PKA

Organotypic hippocampal slices

\begin{abstract}
A B S T R A C T
Thyroid hormone and thyroid hormone metabolites, including 3-iodothyronamine (T1AM) and 3-iodothyroacetic acid (TA1), activate AKT signaling in hippocampal neurons affording protection from excitotoxic damage. We aim to explore whether the mechanism of T1AM neuroprotection against kainic acid (KA)-induced excitotoxicity included the activation of the trace amine associated receptor isoform 1 (TAAR1), one of T1AM targets.

Rat organotypic hippocampal slices were exposed to vehicle (Veh) or to $5 \mu \mathrm{M} \mathrm{kA}$ for $24 \mathrm{~h}$ in the absence or presence of $0.1,1$ and $10 \mu \mathrm{M}$ T1AM or to $0.1,1$ and $10 \mu \mathrm{M}$ T1AM and $1 \mu \mathrm{M}$ N-(3-Ethoxy-phenyl)-4-pyrrolidin-1yl-3-trifluoromethyl-benzamide (EPPTB), the only available TAAR1 antagonist, or to $1 \mu \mathrm{M}$ T1AM in the absence or in the presence of $10 \mu \mathrm{M}$ LY294002, an inhibitor of phosphoinositide 3-kinases (PI3Ks). Cell death was evaluated by measuring propidium iodide (PI) levels of fluorescence $24 \mathrm{~h}$ after treatment. In parallel, the expression levels of p-AKT and p-PKA were evaluated by Western blot analysis of slice lysates. The activity of mitochondrial monoamine oxidases (MAO) was assayed fluorimetrically.

$24 \mathrm{~h}$ exposure of slices to T1AM resulted in the activation of AKT and PKA. KA exposure induced cell death in the CA3 region and significantly reduced p-AKT and p-PKA levels. The presence of 1 and $10 \mu \mathrm{M}$ T1AM significantly protected neurons from death and conserved both kinase levels with the essential role of AKT in neuroprotection. Furthermore, EPPTB prevented T1AM-induced neuroprotection, activation of PKA and AKT. Of note, in the presence of EPPTB T1AM degradation by MAO was reduced.

Our results indicate that the neuroprotection offered by T1AM depends, as for TA1, on AKT activation but do not allow to conclusively indicate TAAR1 as the target implicated.
\end{abstract}

\section{Introduction}

Thyroid hormone and thyroid hormone metabolites, including 3iodothyronamine (T1AM) are endowed of neuroprotective features including memory stimulation and retrieval (Manni et al., 2013; Laurino et al., 2017; Chaalal et al., 2019), reduction of spinal cord injury (Lv et al., 2018; Shulz et al., 2017) and protection of hippocampal neuron cell death induced by kainic-acid (KA; Laurino et al., 2018a). In this latter model we recently reported that thyroid hormone and thyroid hormone metabolites activated hippocampal AKT and that this activation was responsible for the neuroprotective effect of the 3-iodothyroacetic acid (TA1), the main oxidative metabolite of T1AM produced ubiquitously. The neuroprotective mechanism of T1AM at this setting remained instead to be elucidated.

T1AM is the last iodinated thyronamine produced by thyroid hormone alternative metabolism. T1AM, pharmacologically delivered to rodents, presents a short half-life being rapidly degraded to TA1 (Manni et al., 2012) by monoamine oxidases (MAO) or by deiodinases (Laurino

\footnotetext{
Abbreviations: EPPTB, N-(3-Ethoxy-phenyl)-4-pyrrolidin-1-yl-3-trifluoromethyl-benzamide; T1AM, 3-iodothyronamine; TA1, 3-iodothyroacetic acid; MAO, mono-

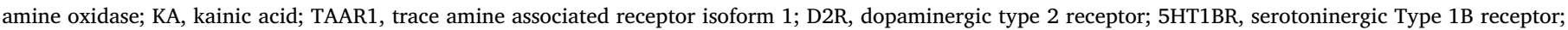
AKT, protein kinase B; PKA, protein kinase A

* Corresponding author. Dept. of NEUROFARBA, Section of Pharmacology, University of Florence, 50139, Florence, Italy.

E-mail address: laura.raimondi@unifi.it (L. Raimondi).

${ }^{1}$ Current address: European Laboratory for Non-Linear Spectroscopy (LENS), University of Florence, 50019 Sesto Fiorentino, Italy.
} 
et al., 2015). Typically, T1AM pharmacological effects onset within $15 \mathrm{~min}$ from amine administration describing inverted dose-effect curves which are modulated by monoamine oxidase inhibitors, thus suggesting that the production of TA1 may be part of T1AM effects (Laurino et al., 2018b) including memory stimulation and hyperalgesia (Manni et al., 2013). Recently, Bellusci et al. (2017) confirmed the participation of TA1 in the T1AM-induced activation of neuroprotective pathways including autophagy. From the pharmacodynamics point of view, T1AM is indicated as a multi-targets molecule able to interact at several G-protein coupled receptors, including the ex-orphan trace amine associated receptor isoform 1 (TAAR1). T1AM is considered an agonist of TAAR1 (Scanlan et al., 2004) but also a biased agonist at dopaminergic type 2 (D2R; Zhang et al., 2018) and serotoninergic type 1B (5HT1BR; Bräunig et al., 2018) receptors which can be found heterodimerized with TAAR1.Indeed, if we exclude the effect of T1AM on the reduction of spinal cord injury (Lv et al., 2018), few of the pharmacological effects reported for T1AM may be attributed unequivocally to TAAR1 activation. Overall, irrespective of which is the plasma membrane receptor recognized, T1AM may also generate, inside cells TA1, a metabolite endowed of an its own signaling capacity and pharmacological activities. Which one of these mechanisms (the plasma membrane and the intracellular ones) prevails could depend on the expression levels of plasma membrane targets and on the amine oxidase cell kit (Laurino et al., 2018b).

In order to get further inside the mechanism of T1AM neuroprotection against excitotoxicity, we aim to verify whether, under the same conditions used to study the neuroprotective effect of TA1, i.e. organotypic hippocampal slices exposed to KA, T1AM resulted protective depending on AKT and if this activation depended on TAAR1 activation.

To this aim, organotypic hippocampal slices were incubated with vehicle or T1AM $(0.1,1$ and $10 \mu \mathrm{M})$ and/or N-(3-Ethoxy-phenyl)-4pyrrolidin-1-yl-3-trifluoromethyl-benzamide (ЕРPTB; $1 \mu \mathrm{M}$ ), the only TAAR1 antagonist commercially available, in absence or presence of $5 \mu \mathrm{M} k \mathrm{k}$. Cell death or the signaling activated by drug exposure was evaluated $24 \mathrm{~h}$ after KA addiction.

\section{Material and methods}

\subsection{Animals}

Male and female Wistar rat pups (7-9 days old) obtained from Charles River (Italy). Animals were housed at $23 \pm 1{ }^{\circ} \mathrm{C}$ under a $12 \mathrm{~h}$ light-dark cycle (lights on at 07:00) and were fed a standard laboratory diet with ad libitum access to water. Experiments and animal use procedures were in accordance with the National Institutes of Health Guide for the Care and Use of Laboratory Animals (NIH Publications No. 8023, revised 1996). The experimental protocols were approved by the ethical Committee of the Italian Council of Health, in compliance with the European Convention for the Protection of Vertebrate Animals used for Experimental and Other Scientific Purposes (ETS no. 123) and the European Communities Council Directive of 24 November 1986 (86/ $609 / \mathrm{EEC}$ ). The authors further attest that all efforts were made to minimize the number of animals used and their suffering.

\subsection{Preparation of rat organotypic hippocampal slice cultures}

Organotypic hippocampal cultures were prepared as previously described (Gerace et al., 2012; Laurino et al., 2018a). Briefly, hippocampi were removed from the brains of Wistar rat pups, transverse slices $(420 \mu \mathrm{m})$ were prepared using a McIlwain tissue chopper and transferred onto 30-mm diameter semi-porous membrane inserts (Millicell-CM PICM03050; Millipore, Italy), which were placed in six-well tissue culture plates containing $1.2 \mathrm{ml}$ of medium per well. The slice culture medium consisted of 50\% Eagle's minimal essential medium, $25 \%$ heat-inactivated horse serum, 25\% Hanks' balanced salt solution, 5
A)

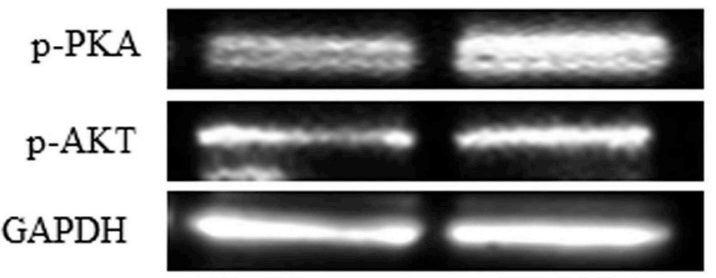

B)
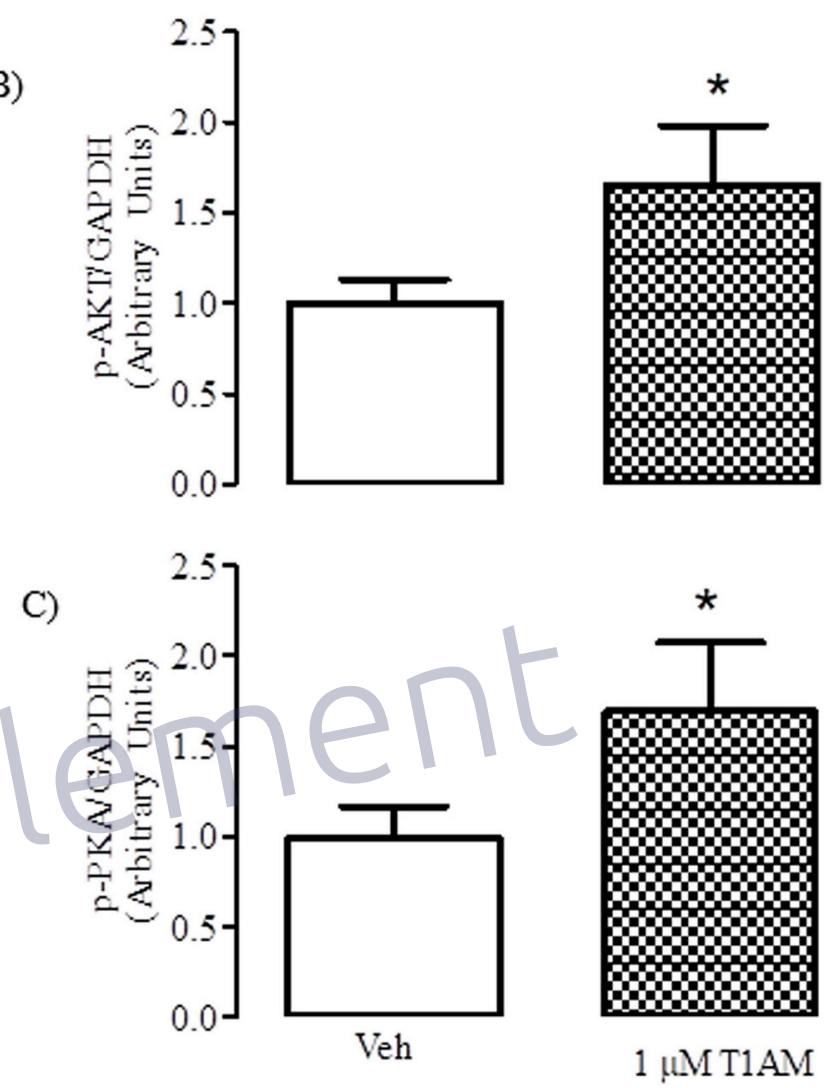

Fig. 1. 3-iodothryonamine (T1AM) activates pro-survival signaling in rat organotypic hippocampal slices.

Rat organotypic hippocampal slices prepared as described in Methods were incubated for $24 \mathrm{~h}$ in the presence of vehicle (Veh) or $1 \mu \mathrm{M}$ T1AM and analyzed for p-AKT and p-PKA levels by Western blot.

A) A representative experiment is shown.

B) Densitometric analysis of p-AKT and p-PKA levels in hippocampal slices. Results are presented as the mean \pm standard error of the mean (SEM) ( $n=4$ for each treatment; * $\mathrm{p}<0.05$ vs. Veh.

$\mathrm{mgml}^{-1}$ glucose, $2 \mathrm{mM}$ L-glutamine, and $3.75 \mathrm{mgml}^{-1}$ amphotericin $\mathrm{B}$. Slices were incubated at $37^{\circ} \mathrm{C}$ in an atmosphere of humidified air and $5 \% \mathrm{CO}_{2}$ for two weeks. Prior to experimentation, all slices were screened for viability by incubating them for $30 \mathrm{~min}$ with the fluorescent dye propidium iodide (PI, $5 \mu \mathrm{gml}^{-1}$; Sigma-Aldrich SRL, Gallarate, Italy). Slices displaying signs of neurodegeneration were excluded from the study.

Slices were exposed to vehicle or $1 \mu \mathrm{M} \mathrm{T} 1 \mathrm{AM}$ for $24 \mathrm{~h}$. At the end of the incubation period, slices were collected for Western blot analysis to evaluate levels of p-AKT, c-fos, p-PKA, p-mTOR, p-GSK-3ß.

\subsection{Cell toxicity evaluation}

This model was utilized under different experimental conditions, as described in the following section. A specific neuronal toxicity in the CA3 region is achieved by exposing slices to $5 \mu \mathrm{M}$ kainic acid (KA) for 
A)

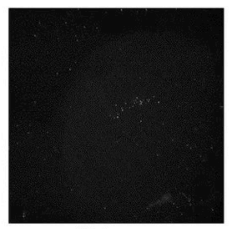

Veh

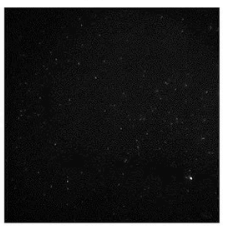

$1 \mu \mathrm{MEPPT}$

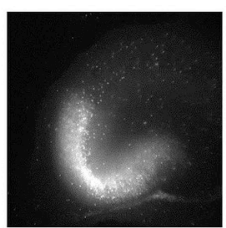

$24 \mathrm{~h} 5 \mu \mathrm{M} \mathrm{KA}$

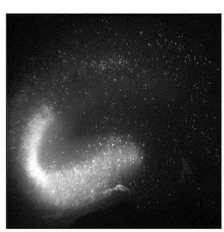

$24 \mathrm{~h} 5 \mu \mathrm{M} \mathrm{KA}+$

$1 \mu \mathrm{M}$ EPPT

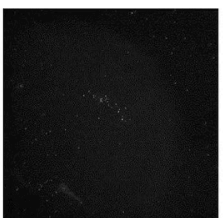

$1 \mu \mathrm{M} \mathrm{T} 1 \mathrm{AM}$

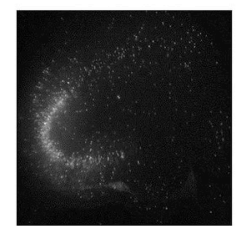

24 h 5 нM KA + $1 \mu \mathrm{M} \mathrm{T} 1 \mathrm{AM}$

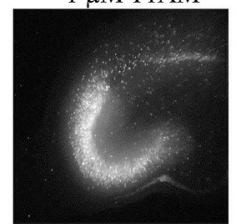

$24 \mathrm{~h} 5 \mu \mathrm{M} \mathrm{KA}+$

$1 \mu \mathrm{MT} 1 \mathrm{AM}+$

$1 \mu \mathrm{MEPPT}$

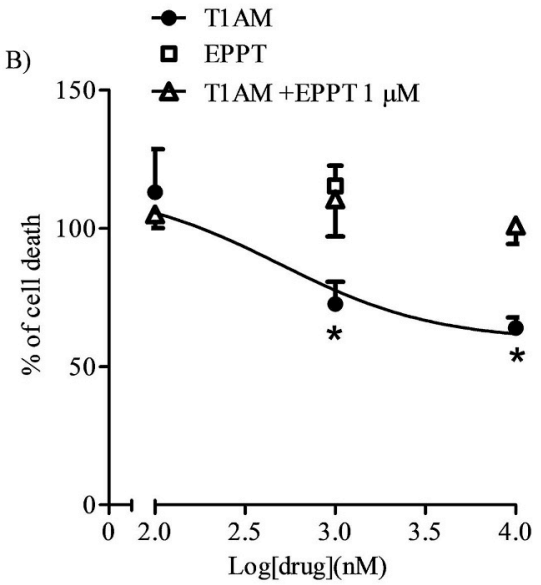

$\Delta$

Fig. 2. 3-iodothryonamine (T1AM) protects against kainic acid (KA) induced neuron cell death depending on AKT activation: the effect of the N-(3-Ethoxyphenyl)-4-pyrrolidin-1-yl-3-trifluoromethyl-benzamide (ЕPPTB).

Organotypic hippocampal slices were exposed to vehicle (Veh) or to $5 \mu \mathrm{M} \mathrm{kA}$ for $24 \mathrm{~h}$ in the absence (Veh) or presence of 0.1-10 $\mu \mathrm{M}$ T1AM or to $1 \mu \mathrm{M}$ EPPTB or 0.1-10 $\mu \mathrm{M}$ T1AM $+1 \mu \mathrm{M}$ EPPTB. In another set of experiments, slices were exposed to $10 \mu \mathrm{M}$ LY294002 (LY) for 15 min before the addition of $1 \mu \mathrm{M}$ T1AM. Cell death was evaluated by measuring propidium iodide (PI) levels of fluorescence $24 \mathrm{~h}$ after treatment. Fluorescence levels measured in hippocampal slices exposed to KA were associated with neuronal death in the CA3 region. Drug effect was reported as a percentage of KA toxicity value (regarded as 100\%). Western blot analysis of hippocampal slices lysates were performed to evaluate the expression levels of p-AKT and p-PKA.

A) Representative experiments of KA-induced cell death are shown.

B) Densitometric evaluation of PI fluorescence in slices exposed to KA (regarded as 100\%) in the absence (Veh) or presence of $0.1-10 \mu \mathrm{M}$ T1AM or 1-10 $\mu \mathrm{M}$ T1AM + $1 \mu \mathrm{M} \mathrm{EPPTB}$

*P $<0.05$ vs. Veh.

Panel C representative experiments of p-AKT and p-PKA activation in hippocampal slices;

Panels D and E : Densitometric analysis of p-AKT and p-PKA levels in hippocampal slices.

Results are presented as the mean \pm standard error of the mean (SEM) $(\mathrm{n}=4$ for each treatment); $* \mathrm{P}<0.05, * * \mathrm{P}<0.01, \mathrm{P}<0.001$ vs. CRL and $\S \mathrm{P}<0.5$ $\S \S \mathrm{P}<0.01$ vs. KA.

Panel F: A representative experiment of KA-induced cell death in the absence and in the presence of LY is shown;

Panel G : Densitometric evaluation of PI fluorescence in slices exposed to KA (regarded as 100\%) in the absence (Veh) or presence of $1 \mu \mathrm{M} \mathrm{T1AM} \mathrm{or} 10 \mathrm{LY}$ or $10 \mathrm{LY}+$ $1 \mu \mathrm{M}$ T1AM; $* * \mathrm{P}<0.01$ vs. Veh.

$24 \mathrm{~h}$ (Laurino et al., 2018a).

Slices were exposed to vehicle or increasing T1AM concentrations $(0.1,1,10 \mu \mathrm{M})$ in the absence or in the presence of $1 \mu \mathrm{M} \mathrm{N}$-(3-Ethoxyphenyl)-4-pyrrolidin-1-yl-3-trifluoromethyl-benzamide (ЕРPTB) and then $5 \mu \mathrm{M} \mathrm{kA}$. In another set of experiments, slices were exposed to $10 \mu \mathrm{M}$ LY294002 (LY), an inhibitor of phosphoinositide 3-kinases (PI3Ks), for $15 \mathrm{~min}$ before the addition of $1 \mu \mathrm{M}$ T1AM.Cell toxicity was evaluated $24 \mathrm{~h}$ later by measuring PI fluorescence (the fluorescence measured in KA-exposed slices in the CA3 region was reported as $100 \%)$.

\subsection{Western blot analysis}

Organotypic hippocampal slices treated as previously described were washed with cold $0.01 \mathrm{M}$ phosphate-buffered saline ( $\mathrm{pH} 7.4$ ), blotted dry, and stored at $-80{ }^{\circ} \mathrm{C}$ until used in Western blot analysis.
Samples were gently transferred and dissolved in a lysis buffer tube containing $50 \mathrm{mM}$ Tris $\mathrm{HCl}$ ( $\mathrm{pH} 8$ ), $150 \mathrm{mM} \mathrm{NaCl}, 1 \mathrm{mM}$ EDTA, $0.1 \% \mathrm{w} /$ $\mathrm{v}$ SDS, and a protease and phosphatase inhibitor cocktail (Thermo Scientific, Monza, Italy). Total protein levels were quantified using the Pierce Protein Assay (Rockford, IL, USA)/BCA (bicinchoninic acid).

Proteins $(20 \mu \mathrm{g})$ isolated from hippocampal slices or from mouse hippocampi were separated via 4-20\% SDS-PAGE and transferred into PVDF membranes (60 min at $398 \mathrm{~mA}$ ) using standard procedures. Blots were incubated overnight at $4{ }^{\circ} \mathrm{C}$ with specific antibodies against p-AKT S473, p-mTOR S2448, p-GSK-3ß S9 (Cell SignalingTechnology, Denver, CO, USA), p-PKA T198 (Santa Cruz Biotechnology Inc. Dallas, TX, USA), and GAPDH (Merck KGaA, Darmstadt, Germany). Primary antibodies were diluted in PBS containing 1\% albumin or 5\% non-fat dry milk and $0.05 \%$ Tween. The antigen-antibody complexes were visualized using appropriate secondary antibodies (1:10 000, diluted in PBS containing $1 \%$ albumin or $5 \%$ non-fat dry milk and $0.05 \%$ Tween) and incubated 


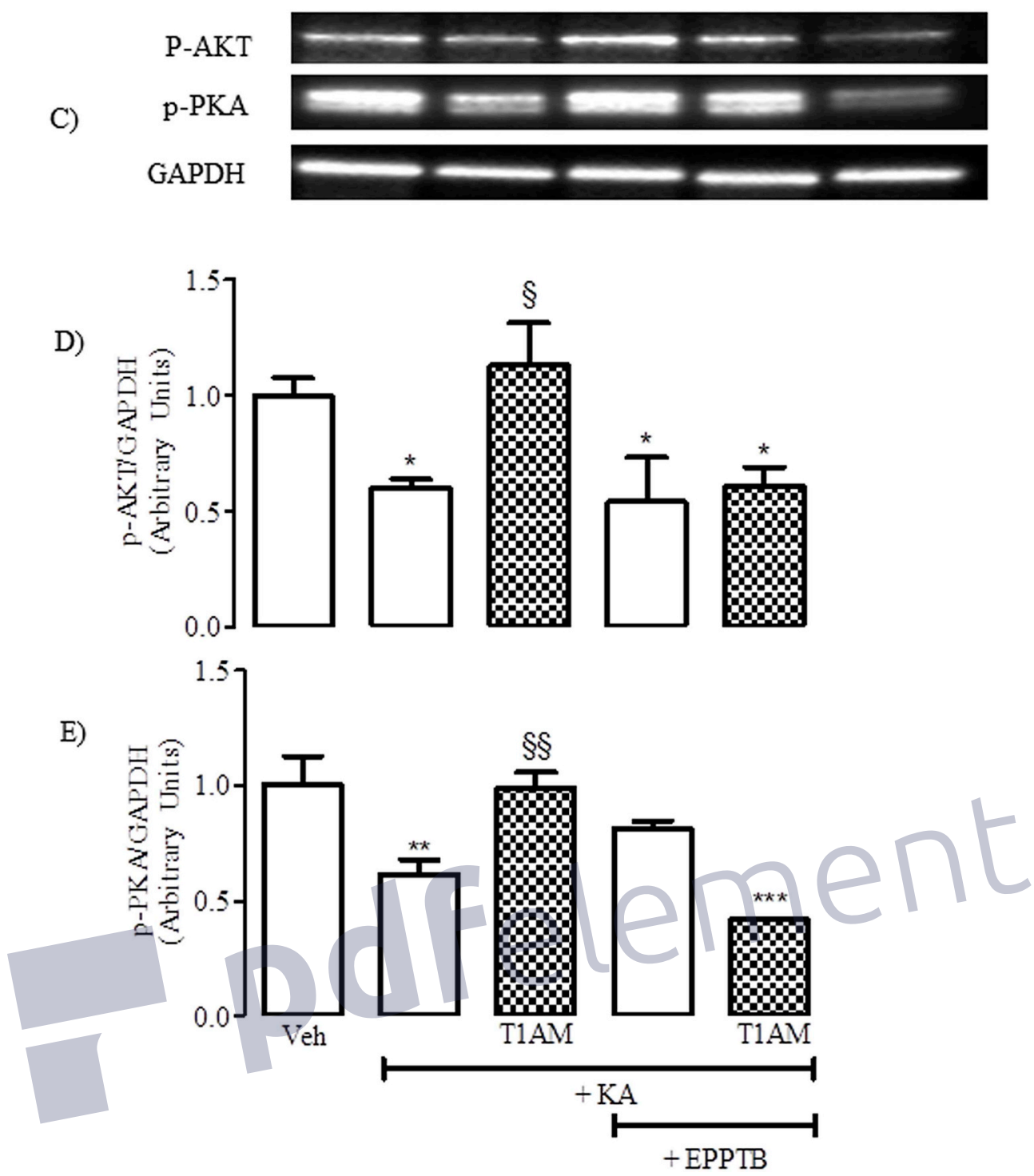

Fig. 2. (continued)

for $1 \mathrm{~h}$ at room temperature. Blots were then extensively washed with PBS containing 0.1\% Tween and developed using an enhanced chemiluminescence detection system (Pierce, Rodano, Italy). Exposition and developing time were standardized for all blots. Densitometric analysis of scanned images was performed on a Macintosh iMac computer using the public domain NIH Image program. Results are presented as the mean \pm SEM of different gels and expressed as arbitrary units (AU), which depict the ratio between levels of target protein expression and GAPDH normalized to basal levels.

EPPTB was a kind gift of Prof. R. Zucchi, University of Pisa, Italy. A stock solution of EPPTB in 100\% DMSO was prepared. The concentration of DMSO never exceeded $0.1 \%$ in our samples.

\subsection{Evaluation of mitochondrial monoamine oxidase (MAO) activity}

Rat hyppocampal slices prepared as described above were homogenized in saline phosphate buffer $\mathrm{pH} 7.4$ and used for the fluorimetric determination of MAO activity (MAO Amplex kit; Sigma Aldrich, Italy). EPPTB $(0.1,1$ and $10 \mu \mathrm{M})$ was pre-incubated for $30 \mathrm{~min}$ at $37^{\circ} \mathrm{C}$ in the presence of enzyme preparations before the addition of $1 \mu \mathrm{M}$ T1AM which was kept in contact with enzymes for $60 \mathrm{~min}$ at the same conditions of temperature. Results were expressed as the mean \pm SEM of Fluorimetric Units/mg of proteins measured in three enzyme preparations with each conditions run in triplicate.

\subsection{Statistical analysis}

Statistical analyses consisted of Unpaired $t$-test or of One or two-way analysis of variance (ANOVA) followed by Dunnet or Bonferroni post hoc test. The threshold of statistical significance was set at $\mathrm{P}<0.05$. Data analysis was performed using GraphPad Prism 5.0 (GraphPad software, San Diego, CA, USA).

\section{Results}

3.1. In rat organotypic hippocampal slices, T1AM induces the activation of $A K T$ and $P K A$

We first investigated whether T1AM, as already described for TA1 (Laurino et al., 2018a), was able to activate an its own signaling including the p-AKT and p-PKA, indicative of the activation of a protective pathway against cell death and of a possible interaction of the amine with a Gs-coupled receptor.

Our results indicated that the exposure of hippocampal slices to $1 \mu \mathrm{M}$ T1AM significantly increased p-AKT and p-PKA levels ( ${ }^{*} \mathrm{P}<0.05$ vs. Veh; Fig. 1, panels A-C). 
F)

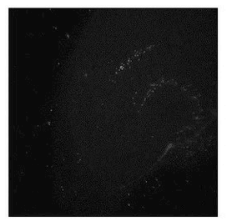

$1 \mu \mathrm{MLY}$

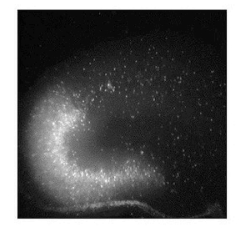

$24 \mathrm{~h} 5 \mu \mathrm{M} \mathrm{KA}$

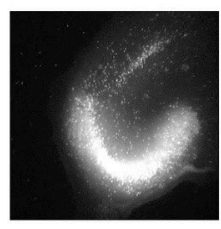

24 h $5 \mu \mathrm{M} \mathrm{KA}+$

$10 \mu \mathrm{M} \mathrm{LY}$

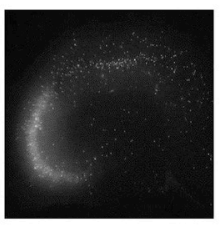

24 h $5 \mu \mathrm{M} \mathrm{KA}+$ $1 \mu \mathrm{M}$ T1AM

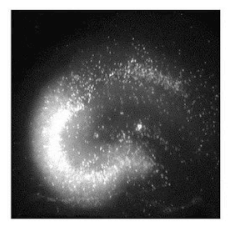

$24 \mathrm{~h} 5 \mu \mathrm{M} \mathrm{KA}+$

$1 \mu \mathrm{M} \mathrm{T} 1 \mathrm{AM}+$ $1 \mu \mathrm{M} \mathrm{LY}$
G)

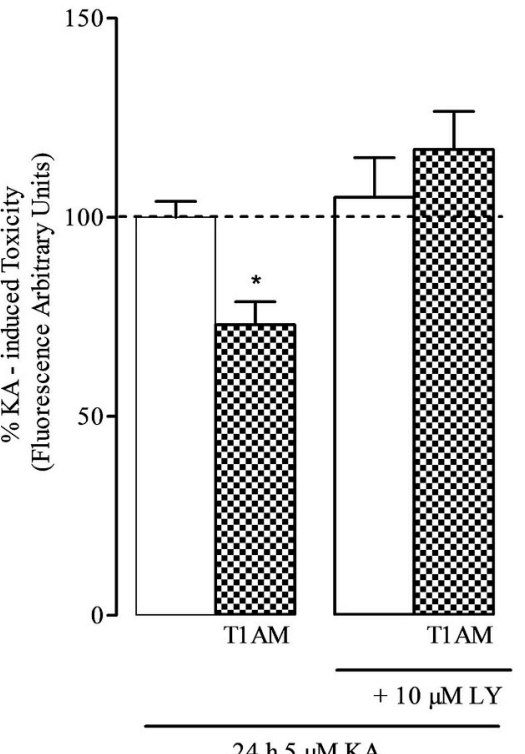

$24 \mathrm{~h} 5 \mu \mathrm{M}$ KA

Fig. 2. (continued)

\subsection{T1AM reduces KA-induced neuronal cell death: the effect of EPPTB}

Slices exposed for $24 \mathrm{~h}$ to $0.1-10 \mu \mathrm{M}$ T1AM did not show any sign of toxicity (Fig. 2, panel A) and KA induced cell death in the CA3 region (Fig. 2, panels A and B). This latter effect associated with a significant reduction of the hippocampal levels of p-AKT and p-PKA (Fig. 2, panels $\mathrm{C}-\mathrm{E} ;{ }^{*} \mathrm{P}<0.05,{ }^{* *} \mathrm{P}<0.01$ vs. Veh). Furthermore, KA-induced cell death was reduced in slices pre-exposed to increasing concentrations of T1AM. The level of protection became significant ( ${ }^{\mathrm{P}}<0.05 \mathrm{vs}$. Veh) in slices exposed to 1 and $10 \mu \mathrm{M}$ T1AM (IC50 $=729 \mathrm{nM}$ ) (Fig. 2, panels $\mathrm{A}$ and $\mathrm{B})$. In parallel, in the presence of $1 \mu \mathrm{M}$ T1AM, the KA-induced reduction of $\mathrm{p}$-AKT and $\mathrm{p}$-PKA $\left({ }^{*} \mathrm{P}<0.05,{ }^{*} \mathrm{P}<0.01\right.$ vs. KA; Fig. 2 , panels C-E) was fully reverted, reporting kinase activation to levels similar to those found in control slices (vehicle treated).

We then verified whether cell death and T1AM signaling were affected by the presence of $1 \mu \mathrm{M}$ EPPTB, a concentration which did not affect cell viability (Fig. 2, panel A). Our results indicated that the presence of $1 \mu \mathrm{M}$ EPPTB completely abolished the protection offered by 1 and $10 \mu \mathrm{M}$ T1AM on KA-induced cell death (Fig. 2, panels A and B). In parallel, hippocampal p-AKT and p-PKA levels were found similar to those measured in KA-treated slices (Fig. 2, panels C-E).

\subsection{T1AM neuroprotective effect depends on the activation of AKT}

To investigate whether the neuroprotection offered by T1AM against KA depended on the activation of AKT, neuronal cell death was

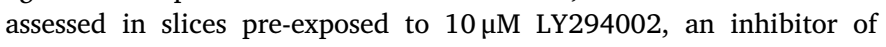
phosphoinositide 3-kinases (PI3Ks; LY) or Veh for $10 \mathrm{~min}$ before the addition of $1 \mu \mathrm{M}$ T1AM. As it is showed in Fig. 2, while $10 \mu \mathrm{M}$ LY did not induce any significant cell toxicity (Fig. 2, panel F), its presence completely prevented the neuroprotection induced by $1 \mu \mathrm{M}$ T1AM (Fig. 2, panels $\mathrm{G}$ and $\mathrm{F}$ ), this result excluding TA1 might participate to T1AM neuroprotection.

\section{4. ЕРPTB inhibits mitochondrial monoamine oxidases (MAO) activity}

We reported previously that, in rat organotypic hippocampal slices, T1AM was rapidly converted into TA1 (Laurino et al., 2015) which is also endowed of neuroprotective effects in vivo and in vitro (Laurino et al., 2018a). To completely exclude any role for TA1 we verified whether TA1 might be really generated at our settings. Our results confirmed that $1 \mu \mathrm{M}$ T1AM was a substrate for MAO but, as a novel finding, we found that T1AM degradation was reduced in the presence of EPPTB [LogIC50 (nM) $4.041 \pm 0.22]$.

\section{Discussion}

We here report that T1AM is an activator of the hippocampal AKT and PKA and that, by activating AKT, T1AM rescues hippocampal neurons from KA-induced excitotoxicity. Both the neuroprotection, the AKT and PKA activation, were prevented by EPPTB, a TAAR1 antagonist, suggesting this receptor is the target controlling AKT activation and then the neuroprotection offered by T1AM. However, since EPPTB is also endowed of MAO inhibitory activity, any role of TA1 in the neuroprotective effect of T1AM, and on AKT activation, cannot be excluded.

T1AM, as already described for TA1 (Laurino et al., 2018a), is able to activate, per se, pro-survival hippocampal pathways including the AKT but also PKA, the kinase coupled to Gs -coupled receptor. This feature is a condition raising the potentiality of cells to survive at negative contests including an excitotoxic attack as that mimicked by exposing hippocampal slices to KA. Consistently, at our settings, KA induced death of neurons of the CA3 region, and, at the same time, reduced the hippocampal levels of p-PKA but also of p-AKT. In the presence of T1AM, KA-induced cell death was reduced and, in parallel, AKT and PKA remained activated. Overall these results supported the existence of a causal relationship between the protection and the activation of these kinases. Consistently, pharmacological evidence indicate that the activation of AKT is essential to determine T1AM-induced neuroprotection. We then wondered whether the activation of PKA and AKT, as well as the neuroprotective effect of T1AM, were secondary to TAAR1 activation. To explore this aim we exposed slices to T1AM in the presence of EPPTB, the only available antagonist of TAAR1. Our results indicate that in the presence of EPPTB, the neuroprotective effect of T1AM, the activation of AKT and of PKA were prevented. These findings fit well with the hypothesis that activation of 
TAAR1 is the only mechanism mediating the neuroprotective effect of T1AM and exclud any role for the TA1 generated inside cells. However, to support this conclusion we verified whether TA1 could be really produced at our settings. Interestingly, our results indicate that EPPTB behaves as an inhibitor of T1AM degradation by MAO. If, from one side, this finding represents an increase of knowledge on the pharmacokinetic features of ЕРPTB, from the other it might mislead the interpretation of results regarding the studies on the pharmacodynamics T1AM. In particular, in the context of the mechanism of T1AM-induced neuroprotection here studied, the fact that EPPTB inhibits MAO activity does not allow to conclusively exclude the possibility that the activation of AKT found in slices exposed to T1 AM might derive, at least in part, from the intracellular production of TA1 rather than from TAAR1 activation.

\section{Conclusions}

T1AM, as TA1, may be consider as an endogenous activator of prosurvival mechanisms increasing neuron defenses against excitotoxicity with a mechanism which remains to be conclusively addressed. However, the capacity of T1AM, to activate AKT further supports a role of thyroid secretome in neuroprotection.

\section{Conflicts of interest}

All the authors have not any conflict of interest to declare.

\section{Funding}

This paper was supported by a grant from the University of Florence and by Ente Cassa di Risparmio di Firenze.

\section{Appendix A. Supplementary data}

Supplementary data to this article can be found online at https:// doi.org/10.1016/j.neuint.2019.05.004.

\section{References}

Bellusci, L., Laurino, A., Sabatini, M., Sestito, S., Lenzi, P., Raimondi, L., Rapposelli, S., Biagioni, F., Fornai, F., Salvetti, A., Rossi, L., Zucchi, R., Chiellini, G., 2017. New insights into the potential roles of 3-iodothyronamine (T1AM) and newly developed thyronamine-like TAAR1 agonists in neuroprotection. Front. Pharmacol. 12 (8), 905. https://doi.org/10.3389/fphar.2017.00905. eCollection 2017.

Bräunig, J., Dinter, J., Höfig, C.S., Paisdzior, S., Szczepek, M., Scheerer, P., Rosowski, M., Mittag, J., Kleinau, G., Biebermann, H., 2018. The trace amine-associated receptor 1 agonist 3-iodothyronamine induces biased signaling at the serotonin $1 \mathrm{~b}$ receptor. Front. Pharmacol. 12 (9), 222. https://doi.org/10.3389/fphar.2018.00222. eCollection 2018.

Chaalal, A., Poirier, R., Blum, D., Laroche, S., Enderlin, V., 2019. Thyroid hormone supplementation restores spatial memory, hippocampal markers of neuroinflammation, plasticity-related signaling molecules, and $\beta$-amyloid peptide load in hypothyroid rats. Mol. Neurobiol. 56, 722-735. https://doi.org/10.1007/s12035-0181111-z. Epub 2018 May 23.

Gerace, E., Landucci, E., Scartabelli, T., Moroni, F., Pellegrini-Giampietro, D.E., 2012. Rat hippocampal slice culture models for the evaluation of neuroprotective agents. Methods Mol. Biol. 846, 343-354. https://doi.org/10.1007/978-1-61779-536-7_29.

Laurino, A., De Siena, G., Saba, A., Chiellini, G., Landucci, E., Zucchi, R., Raimondi, L., 2015. In the brain of mice, 3-iodothyronamine (T1AM) is converted into 3-iodothyroacetic acid (TA1) and it is included within the signaling network connecting thyroid hormone metabolites with histamine. 761:130-4. Eur. J. Pharmacol. 15https://doi.org/10.1016/j.ejphar.2015.04.038. Epub 2015 May 1.

Laurino, A., Landucci, E., Resta, F., De Siena, G., Pellegrini-Giampietro, D.E., Masi, A., Mannaioni, G., Raimondi, L., 2018a. Anticonvulsant and neuroprotective effects of the thyroid hormone metabolite 3-iodothyroacetic acid. Thyroid 28, 1387-1397.

Laurino, A., Landucci, E., Raimondi, L., 2018b. Central effects of 3-iodothyronamine reveal a novel role for mitochondrial monoamine oxidases. Front. Endocrinol. 9, 290 https://doi.org/10.3389/fendo.2018.00290. eCollection 2018. (submitted for publication).

Laurino, A., Lucenteforte, E., De Siena, G., Raimondi, L., 2017. The impact of scopolamine pretreatment on 3-iodothyronamine (T1AM) effects on memory and pain in mice. Horm. Behav. 94, 93-96.

Lv, J., Liao, J., Tan, W., Yang, L., Shi, X., Zhang, H., Chen, L., Wang, S., Li, Q., 2018. 3Iodothyronamine acting through an anti-apoptotic mechanism is neuroprotective against spinal cord injury in rats. Ann. Clin. Lab. Sci. 48, 736-742.

Manni, M.E., De Siena, G., Saba, A., Marchini, M., Dicembrini, I., Bigagli, E., Cinci, L., Lodovici, M., Chiellini, G., Zucchi, R., Raimondi, L., 2012. 3-Iodothyronamine: a modulator of the hypothalamus-pancreas-thyroid axes in mice. Br. J. Pharmacol. 166, 650-658. https://doi.org/10.1111/j.1476-5381.2011.01823.

Manni, M.E., De Siena, G., Saba, A., Saba, A., Marchini, M., Landucci, E., Gerace, E., Zazzeri, M., Musilli, C., Pellegrini-Giampietro, D., Matucci, R., Zucchi, R., Raimondi, L., 2013. Pharmacological effects of 3-iodothyronamine (T1AM) in mice include facilitation of memory acquisition and retention and reduction of pain threshold. Br. J. Pharmacol. 168, 354-362. https://doi.org/10.1111/j.1476-5381.2012.02137.x.

canlan, T.S., Suchland, K.L., Hart, M.E., Chiellini, G., Huang, Y., Kruzich, P.J., Frascarelli, S., Crossley, D.A., Bunzow, J.R., Ronca-Testoni, S., Lin, E.T., Hatton, D., Zucchi, R., Grandy, D.K., 2004. 3-Iodothyronamine is an endogenous and rapidacting derivative of thyroid hormone. Nat. Med. 10, 638-642 Epub 2004 May 16.

Shultz, R.B., Wang, Z., Nong, J., Zhang, Z., Zhong, Y., 2017. Local delivery of thyroid hormone enhances oligodendrogenesis and myelination after spinal cord injury. J. Neural Eng. 14, 036014. https://doi.org/10.1088/1741-2552/aa6450. Epub 2017 Mar 30.

Zhang, X., Mantas, I., Alvarsson, A., Yoshitake, T., Shariatgorji, M., Pereira, M., Nilsson, A., Kehr, J., Andrén, P.E., Millan, M.J., Chergui, K., Svenningsson, P., 2018. Striata tyrosine hydroxylase is stimulated via TAAR1 by 3-iodothyronamine, but not by tyramine or $\beta$-phenylethylamine. Front. Pharmacol. 9, 166. https://doi.org/10.3389/ fphar.2018.00166. eCollection 201. 